\title{
Advances in Gastrointestinal Nutrient Sensor Research: Nutritional Pharmacy for Elderly Health Fundamentals
}

\author{
Hisayuki UNEYAMA ${ }^{*, a}$ and Koji TAKEUCHI ${ }^{b}$ \\ aUmami Wellness Research Group, Frontier Research Labs., Institute for Innovation, Ajinomoto Co., Inc., \\ 1-1, Suzuki-cho, Kawasaki-Ku, Kawasaki 210-8681, Japan, and bivision of Pathological Sciences, \\ Department of Pharmacology and Experimental Therapeutics, Kyoto Pharmaceutical \\ University, Misasagi, Yamashina, Kyoto 607-8414, Japan
}

This special chapter summarizes the contents of the symposium entitled "Advances in Gastrointestinal Nutrient Sensor Research: Nutritional Pharmacy for Elderly Health Fundamentals" that was scheduled during the 131st annual meeting of the Pharmaceutical Society of Japan.

Recent advances in taste physiology have revealed a variety of taste receptors such as umami (T1R/T1R3 and mGluRs), sweet (T1R/T1R3) and fat tastes (GPR120), all of which recognize nutrients within the oral cavity. These receptors are widely distributed in the gastrointestinal tract and mediate luminal nutrient information to the brain for regulating the body nutrient homeostasis. ${ }^{1,2}$ The gut nutrient perception affects a variety of gastrointestinal functions such as motility and exocrine (saliva, gastric and pancreatic juices) as well as endocrine (insulin, CCK, ghrelin et al.) secretion via neuronal and hormonal pathways. The gut chemical senses are particularly important in the formation of eating habits and the maintenance of gut function. Eastern and Western countries that have aging populations need to resolve problems that prevent the elderly from living a healthy life. The elderly can be at risk for under-nutrition due to gastrointestinal troubles such as swallowing disorders, dyspepsia and constipation. Improvement of gastrointestinal functions will help the elderly live healthy lives.

In this symposium, we invited five distinct experts with advanced research specialties in taste and nutrition in addition to the organizers. First, Dr. Takeda of Tokushima University described the patho-physiology and diagnosis of taste disorders in clinics. ${ }^{3)}$ Second, Dr. Ebihara at Tohoku University proposed a

\footnotetext{
*e-mail: hisayuki_uneyama@ajinomoto.com

This article is based on the foreword for Symposia 15

(S15) of the 131st Annual Meeting of the Pharmaceutical

Society of Japan.
}

new clinical therapeutic strategy for swallowing dysfunction and aspiratory pneumonia with management of oral chemical sensing. ${ }^{4)}$ From the basic research field, we focused on recent advances in gut glutamate research (Dr. Uneyama of Ajinomoto Co., Inc. and Dr. Amagase of Kyoto Pharmaceutical University), ${ }^{5,6)}$ and fatty acid research (Dr. Hirasawa of Kyoto University and Dr. Karaki of Shizuoka Prefectural University. ${ }^{7,8)}$

The present approach by focusing on taste and gastrointestinal nutrient-sensing might provide clues for improving the nutritional status and QOL of the elderly. We also believe that it is necessary to establish a new pharmaceutical research field in nutritional pharmacy, which combines nutrition and pharmacy for treatment of human diseases.

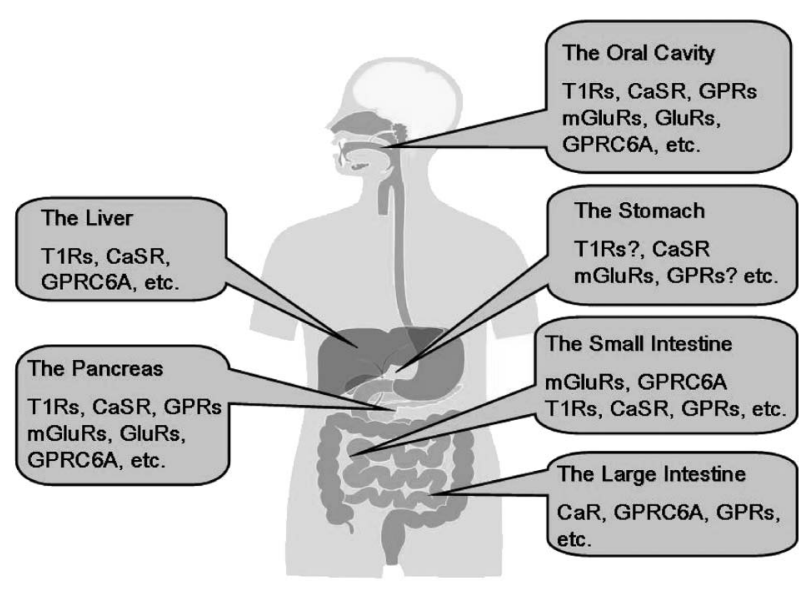

Fig. 1. Distribution of Nutrient Sensors in Gastrointestinal Organs

Summary of current knowledge on the taste receptor gene expression is illustrated. T1Rs; T1R1/T1R3 (umami taste), T1R1/T1R3 (sweet taste); mGluRs; metabotrophic glutamate receptors; mGluR1 and mGluR4 (umami taste); CaSR; calcium-sensing receptor (kokumi taste), GluR; ionotrophic glutamate receptors; NMDA and KA receptors (umami taste); GPRs; GPR 40, 41, 120 (fatty acids); GPRC6A (lysine and arginine). 


\section{REFERENCES}

1) Uneyama H., Tanaka T., Torii K., Nippon Yakurigaku Zasshi, 124, 210-218 (2004).

2) Berthould H., Regul. Pept., 149, 15-25 (2008).

3) Takaoka T., Sarukura N., Ueda C., Kitamura Y., Kalubi B., Toda N., Abe K., Yamamoto S., Takeda N., Auris Nasus Larynx, 37, 190194 (2010).

4) Ebihara S., Kohzuki M., Sumi Y., Ebihara T., J. Pharmacol. Sci., 115, 99-104 (2011).
5) Uneyama H., San Gabriel A., Kawai M., Tomoe M., Torii K., Asia Pac. J. Clin. Nutr., 17, 372-375 (2008).

6) Amagase K., Ochi A., Kojo A., Mizunoe A., Taue M., Kinoshita N., Nakamura E., Takeuchi K., J. Pharmacol. Sci. (2011). (in press)

7) Hara T., Hirasawa A., Ichimura A., Kimura I., Tsujimoto G., J. Pharm. Sci., 100, 35943601 (2011).

8) Tazoe H., Otomo Y., Karaki S., Kato I., Fukami Y., Terasaki M., Kuwahara A., Biomed. Res., 30, 149-156 (2009). 